\title{
Cohort study on 20 years' experience of bilateral video- assisted thoracic sympathectomy (VATS) for treatment of hyperhidrosis in 2431 patients
}

\author{
Nelson Wolosker', José Ribas Milanez de Campos", Paulo Kauffman"', Marcelo Fiorelli Alexandrino da Silva'", Carolina Brito
} Faustinov", Miguel Lia Tedde ${ }^{v \prime}$, Pedro Puech-Leãov"I, Paulo Manuel Pêgo Fernandes ${ }^{\text {VIII }}$

Faculdade de Medicina da Universidade de Sao Paulo and Hospital Israelita Albert Einstein, São Paulo (SP), Brazil

'MD, PhD. Full Professor, Hospital Israelita Albert Einstein (HIAE), São Paulo (SP), Brazil; and Surgeon, Department of Surgery, Vascular and Endovascular Division, Faculdade de Medicina, Universidade de Sao Paulo, Sao Paulo, SP, BR.

(D) https://orcid.org/0000-0003-1991-3507

"MD, PhD. Surgeon, Hospital Israelita Albert Einstein (HIAE), São Paulo (SP), Brazil; and Surgeon, Department of Surgery, Thoracic Surgery Division, Faculdade de Medicina, Universidade de Sao Paulo, Sao Paulo, SP, BR. (D) https://orcid.org/0000-0002-2385-7707

"'MD, PhD. Surgeon, Hospital Israelita Albert Einstein (HIAE), São Paulo (SP), Brazil; and Surgeon, Department of Surgery, Vascular and Endovascular Division, Faculdade de Medicina, Universidade de Sao Paulo, Sao Paulo, SP, BR.

(D) https://orcid.org/0000-0002-4363-4148

"MD. Surgeon, Hospital Israelita Albert Einstein (HIAE), São Paulo (SP), Brazil; and Surgeon, Department of Surgery, Vascular and Endovascular Division, Faculdade de Medicina, Universidade de Sao Paulo, Sao Paulo, SP, BR. (D) https://orcid.org/0000-0002-0714-5291

'MD. Surgeon, Hospital Israelita Albert Einstein (HIAE), São Paulo (SP), Brazil; and Surgeon, Department of Surgery, Vascular and Endovascular Division, Faculdade de Medicina, Universidade de Sao Paulo, Sao Paulo, SP, BR

(D) https://orcid.br/0000-0002-4858-9796

"MD, PhD. Surgeon, Department of Surgery, Thoracic Surgery Division, Faculdade de Medicina, Universidade de Sao Paulo, Sao Paulo, SP, BR

(D) https://orcid.org/0000-0002-8178-4243

"IIMD, PhD. Surgeon, Department of Surgery, Vascula and Endovascular Division, Faculdade de Medicina, Universidade de Sao Paulo, Sao Paulo, SP, BR.

(D) https://orcid.org/0000-0002-6974-9509

VIIIMD, PhD. Full Professor, Thoracic Surgery Program, Instituto do Coração (InCor), Hospital das Clínicas (HC), Faculdade de Medicina da Universidade de São Paulo (FMUSP), São Paulo (SP), Brazil; and Cardiothoracic

Surgeon, Hospital Beneficência Portuguesa (BP), São Paulo (SP), Brazil.

(D) https://orcid.org/0000-0001-7243-5343

KEY WORDS (MeSH terms):

Hyperhidrosis.

Quality of life.

Thoracoscopy.

AUTHORS' KEY WORDS:

Sympathotomy.

VATS.

Excessive sweating

\section{ABSTRACT}

BACKGROUND: Primary hyperhidrosis is a condition characterized by excessive sweating, inconsistent with the needs for thermoregulation.

OBJECTIVE: To assess the effectiveness and the change in the quality of life of patients undergoing bilateral VATS (video-assisted thoracoscopic sympathectomy) for treatment of hyperhidrosis, in a large case series.

DESIGN AND SETTING: Cohort study conducted in a tertiary hospital specializing in hyperhidrosis located in São Paulo, Brazil.

METHODS: A total of 2,431 patients who underwent surgery consisting of bilateral video-assisted thoracoscopic sympathectomy between January 2000 and February 2017 were retrospectively assessed in an outpatient clinic specializing in hyperhidrosis. The patients underwent clinical and quality of life assessments on two occasions: firstly, prior to surgery, and subsequently, one month after the operation. The presence or absence of compensatory hyperhidrosis $(\mathrm{CH})$ and general satisfaction after the first postoperative month were also evaluated.

RESULTS: All the patients operated had poor or very poor quality of life before surgery. In the postoperative period, an improvement in the quality of life was observed in more than $90 \%$ of the patients. Only $10.7 \%$ of the patients did not present $\mathrm{CH}$, and severe $\mathrm{CH}$ occurred in $22.1 \%$ of the patients in this sample. CONCLUSIONS: Bilateral VATS is a therapeutic method that decreases the degree of sweating more than $90 \%$ of patients with palmar and axillary hyperhidrosis. It improves the quality of life for more than $90 \%$ of the patients, at the expense of development of $\mathrm{CH}$ in approximately $90 \%$ of the patients, but not intensely.

\section{INTRODUCTION}

Primary hyperhidrosis $(\mathrm{PH})$ is a condition characterized by excessive sweating that is inconsistent with thermoregulation needs. It has a large impact on patients' quality of life and affects their personal and professional relationships. ${ }^{1,2}$ In the majority of cases, $\mathrm{PH}$ manifests in childhood and adolescence and persists throughout life. The typical clinical presentation is limited to the palms of the hands, the plantar region of the foot and/or the axilla, and it is symmetrical. It can also affect the head and face and often occurs in two or more regions of the body. The pathophysiology of $\mathrm{PH}$ is not fully understood, but it is known to result from stimulation of the sympathetic nervous system in its regulatory center. $\mathrm{PH}$ affects approximately $2.8 \%$ of the population, and there is a positive family history in $12.5 \%$ to $56.5 \%$ of the patients. ${ }^{3}$ Patients generally seek medical care later in life, and more frequently at a more financially secure age. Thus, young people end up suffering for many years before being able to receive the current well-known medical treatment.,

The initial treatment for patients with PH, until 2010, was sympathectomy. Thereafter, we began to use oxybutynin chloride as the first-line treatment. In patients for whom no adequate response to medication is attained, video-assisted thoracoscopic sympathectomy (VATS) becomes the treatment of choice.

VATS is considered to be the gold standard for the definitive treatment of hyperhidrosis. It provides excellent clinical results (reduced sweating at specific sites) and leads to a significant improvement in quality of life. These positive results are, among other causes, based on factors that are known to influence the effectiveness of sympathectomy among patients with hyperhidrosis, ${ }^{1}$ such as body mass index, resection level, ${ }^{6,7}$ preoperative quality of life $^{8}$ and the number of resected ganglia. ${ }^{9}$ 


\section{OBJECTIVE}

The objective of this study was to assess the effectiveness of the treatment and the change in the quality of life of patients undergoing VATS in a large case series (2,431 patients).

\section{METHODS}

A total of 2,431 patients who underwent bilateral video-assisted thoracoscopic sympathectomy between January 2000 and February 2017 were retrospectively assessed in an outpatient clinic specializing in hyperhidrosis. This study was approved by the research ethics committee of our institution (protocol number: 1.133.255; date: June 30, 2015).

All patients treated under our care since 2000 have been routinely evaluated through regular completion of a specific medical record that includes demographic data and description of all sites of hyperhidrosis and the quantitative intensity of hyperhidrosis complaints at each site, using a questionnaire (HDSS). This is a specific quality-of-life questionnaire that was standardized by Amir et al. and translated into English by Campos et al. The quantitative degree of improvement at each point after treatment is assessed using this specific questionnaire. ${ }^{10,11}$

Regarding the surgical technique, in general terms, the surgery is performed with the patient in a semi-Fowler's position at $45^{\circ}$ to the floor. Two small incisions of approximately $1.0 \mathrm{~cm}$ are made in each hemithorax. The pleural cavity is accessed through an incision that is made in the fourth intercostal space, on the anterior axillary line. Through this, a $5 \mathrm{~mm}$ and $30^{\circ}$ video optic is introduced. The second incision is made in the second intercostal space, on the middle axillary line, for insertion of an endoscopic scalpel. After identification of the sympathetic chain, the ganglion is isolated, always starting from the medial costal pleura, following the lateral costal pleura and ending with complete dissection of the sympathetic chain and the ganglion.

The sympathetic chain is resected on the respective costal arches. At the end of this step, the sympathetic chain segment located between the corresponding costal arches, including the target ganglion, is electrocauterized. During the sympathectomy, all patients are kept temporarily in apnea or low-flow ventilation. At the end of the surgery, the residual pneumothorax is aspirated through a nasogastric tube (no. 16) and pulmonary expansion is monitored via the video optic. The incisions are closed with intradermal sutures.

Anatomically, sympathectomy performed on the right side of the chest is slightly more laborious than on the left side owing to the greater number of large-caliber veins on the thoracic sympathetic chain and its more superior branches. This forces the surgeon to be more careful in the dissection.

Patients are usually extubated without difficulty in the operating room. After awakening from anesthesia, they are sent for anesthetic recovery and then to their room. To ensure complete pulmonary reexpansion, chest radiographs are routinely performed shortly after the surgery.

The patients underwent clinical and quality of life assessments on two occasions: firstly, prior to surgery, and subsequently, one month after the operation. All the evaluations were done by the primary investigator. The primary endpoints in the study were the following:

1. The patients' quality of life at the first visit prior to surgery.

2. The improvement in quality of life after surgery, the clinical improvement in sweating, the presence or absence of compensatory hyperhidrosis $(\mathrm{CH})$ and general satisfaction after the first postoperative month.

To measuring the degree of satisfaction, we used the quality-of-life protocol described by Amir et al., which was translated to English by Campos et al. ${ }^{10-12}$ Before the surgical treatment, the patients completed quality-of-life assessments without physician involvement. This protocol consists of 20 questions divided into four domains (functional-social, personal, emotional and special conditions). Five response levels are described in tables, from which only one response is allowed for each question. The patients were classified into five different levels of satisfaction, calculated as the summed total score from the protocol, ranging from 20 to 100 . When the sum was greater than 84, the quality of life was considered very poor; from 69 to 83 , poor; from 52 to 68 , good; from 36 to 51 , very good and from 20 to 35 , excellent.

The improvement in quality of life after surgery was evaluated using the same protocol, and the patients were classified into five different levels of improvement, calculated as the total score from the protocol. For scores greater than 84 , the quality of life was considered much worse after the surgical treatment; from 69 to 83 , a little worse; from 52 to 68 , equal; from 36 to 51 , a little better; and from 20 to 35 , much better.

The clinical improvement in sweating after treatment was defined on a quantitative scale ranging from 0 to 10 , in which 0 represented no improvement and 10 represented absence of sweat, or anhidrosis, for each site of previous hyperhidrosis, based on the patients' own assessments. From this score regarding the patients' main complaint, the clinical improvement was graded as follows: null, 0-4; moderate, 5-7; or good, 8-10.

The degree of patient satisfaction after surgery was quantified using a questionnaire with four options for patients to describe their general satisfaction with surgery outcomes. The patients' general satisfaction was considered to be excellent if they were $100 \%$ satisfied with the surgery outcome one month after surgery, good if they were $90 \%$ satisfied, fair if they were $75 \%$ satisfied or low if they were less than $50 \%$ satisfied. 
The patients' reports, with confirmation through physical examination, were used to analyze the incidence of $\mathrm{CH}$. The severity of $\mathrm{CH}$ was graded as severe or non-severe. $\mathrm{CH}$ was considered to be severe if it was visible and embarrassing, and required more than one change of clothes during the day. $\mathrm{CH}$ was considered to be non-severe if it was visible and embarrassing but not enough to require a change of clothes, or if it was visible and embarrassing only sometimes (e.g. in hot weather and during exercise), or if it was present but did not bother the patient.

\section{RESULTS}

The demographic data, main site of hyperhidrosis and the level of VATS resection in the group are presented in Table 1 . The prevalence was higher among females. The mean age of the group was 24.7 years and the mean body mass index was $21.7 \mathrm{~kg} / \mathrm{m}^{2}$. The main sites of hyperhidrosis were palmar and axillary. Resection of a single G3 ganglion was the most frequent procedure, followed by resection of a $\mathrm{G} 4$ ganglion.

The assessment of quality of life before surgery and the improvement in quality of life after surgery among the patients are shown in Table 2. It should be noted that all the patients operated under our care had poor or very poor quality of life before surgery. In the postoperative period, an improvement in the quality of life was observed in more than $90 \%$ of the patients.

A review of the clinical improvement at the main site of sweating after surgery is presented in Table 3. At the palmar and axillary sites that were the main site of hyperhidrosis, more than $90 \%$ of the patients in this study reported major clinical improvement.

Table 1. Demographic and technical data

\begin{tabular}{|c|c|c|c|}
\hline & \multirow{2}{*}{ Variable } & \multicolumn{2}{|c|}{ Patients } \\
\hline & & \multicolumn{2}{|c|}{$(n=2431)$} \\
\hline \multirow{2}{*}{ Sex (n \%) } & Female & 1,618 & $67.4 \%$ \\
\hline & Male & 783 & $32.6 \%$ \\
\hline Age (years) & Mean/SD & 24.7 & 7.57 \\
\hline BMI $\left(\mathrm{kg} / \mathrm{m}^{2}\right)$ & Mean/SD & 21.7 & 2.80 \\
\hline \multirow{4}{*}{ Main site ( $\mathrm{n} \%$ ) } & Palmar & 1,546 & $64.4 \%$ \\
\hline & Axillary & 785 & $32.7 \%$ \\
\hline & Plantar & 29 & $1.2 \%$ \\
\hline & Cranial-facial & 42 & $1.7 \%$ \\
\hline \multirow{7}{*}{ Technique (n \%) } & $\mathrm{G} 2$ & 100 & $4.2 \%$ \\
\hline & G2/G3 & 365 & $15.2 \%$ \\
\hline & G2/G3/G4 & 1 & $0.0 \%$ \\
\hline & G3 & 838 & $35.0 \%$ \\
\hline & $\mathrm{G} 3 / \mathrm{G} 4$ & 264 & $11.0 \%$ \\
\hline & G4 & 828 & $34.5 \%$ \\
\hline & G4/G5 & 0 & $0.0 \%$ \\
\hline
\end{tabular}

$\mathrm{BMI}=$ body mass index; $\mathrm{SD}=$ standard deviation; $\mathrm{G}=$ ganglion .
The rate of improvement was lower (80.6\%) in cases in which the cranial-facial region was the main site.

An analysis on the prevalence and intensity of $\mathrm{CH}$ is presented in Table 4 . Only $10.7 \%$ of the patients did not present $\mathrm{CH}$, and severe $\mathrm{CH}$ occurred in $22.1 \%$ of the patients in this sample.

An analysis on the degree of satisfaction among the patients after surgery in both groups is shown in Table 5. More than $90 \%$ of the patients reported having high satisfaction with the surgery.

A correlation between the technique used and presence of compensatory hyperhidrosis is shown in Table 6. Ablation at lower levels (especially G4) resulted in a lesser degree of compensatory hyperhidrosis $(\mathrm{P}<0.00001)$.

Table 2. Reported pre and postoperative quality of life over the age range of the patients

\begin{tabular}{lcc|} 
& & Patient group \\
& & $\mathbf{n}=\mathbf{2 , 4 3 1}$ \\
& Excellent & 0 \\
Preoperative QoL & Very good & 0 \\
{$[\mathrm{n}(\%)]$} & Good & 0 \\
& Poor & $630(28.0 \%)$ \\
& Very poor & $1617(72.0 \%)$ \\
\hline Postoperative QoL & Much better & $1,566(78.6 \%)$ \\
{$[n(\%)]$} & A little better & $321(16.1 \%)$ \\
& Equal & $76(3.8 \%)$ \\
& A little worse & $19(0.9 \%)$ \\
& Much worse & $10(0.5 \%)$
\end{tabular}

QoL = quality of life.

Table 3. Analysis on clinical improvement after surgery, at the main site of hyperhidrosis (palmar, axillary or cranial-facial)

$\begin{array}{lcc}\text { Main site of PH } & \begin{array}{c}\text { Degree of clinical } \\ \text { improvement }\end{array} & \begin{array}{c}\text { Patients } \\ \mathbf{n}=\mathbf{2 4 3 1}\end{array} \\ \text { Palmar } & \text { High } & 1,465(94.4 \%) \\ \mathrm{n}=1,552 & \text { Moderate } & 75(4.8 \%) \\ & \text { Null } & 12(0.8 \%) \\ \text { Axillary } & \text { High } & 692(90.5 \%) \\ \mathrm{n}=765 & \text { Moderate } & 59(7.7 \%) \\ & \text { Null } & 14(1.8 \%) \\ \text { Cranial-facial } & \text { High } & 29(80.6 \%) \\ \mathrm{n}=36 & \text { Moderate } & 6(16.7 \%) \\ & \text { Null } & 1(2.8 \%)\end{array}$

$\mathrm{PH}=$ primary hyperhidrosis

Table 4. Prevalence of compensatory hyperhidrosis and its intensity

$\begin{array}{lcc} & \text { Patients } \\ & & \mathbf{n}=\mathbf{2 , 3 6 2} \\ \text { Compensatory } & \text { Absent } & 251(10.7 \%) \\ \text { hyperhidrosis } & \text { Non-severe } & 1,588(67.2 \%) \\ & \text { Severe } & 523(22.1 \%)\end{array}$




\section{DISCUSSION}

Primary hyperhidrosis is a disease that significantly affects the population, and in particular the younger population. These patients seek medical evaluations and treatments to achieve a general improvement in their quality of life. ${ }^{13}$

Currently in our practice, we start treatment with oxybutynin hydrochloride in all patients. ${ }^{14-16}$ This medication was proven to be effective as an initial therapy in a randomized, placebo-controlled trial by Wolosker et al., in 2012. Positive results were obtained over both the short and the long term, while the quality of life remained unchanged in situations of treatment failure. ${ }^{17}$ In such cases, surgical treatment was considered. ${ }^{16,18}$

Epidemiological evaluation of $\mathrm{PH}$ has revealed that there is high demand for care from young adults. It has been demonstrated that the prevalence of primary hyperhidrosis in the general population is equal between sexes. ${ }^{19,20}$ However, there is greater demand for treatment from females, which is due, among other reasons, to greater concern for esthetics in this group. ${ }^{20,21}$ This was shown in our previous study in which $67.4 \%$ of the patients were female. ${ }^{22}$

In our practice, we only operate on patients with a body mass index (BMI) lower than 25, since greater prevalence of severe $\mathrm{CH}$ has been observed among patients with a BMI greater than 25 who underwent the operation. ${ }^{23}$ This is why the mean BMI was 21.7 $\mathrm{kg} / \mathrm{m}^{2}$ in the sample of our previous study. ${ }^{22}$

Regarding the distribution of the main hyperhidrosis sites, palmar hyperhidrosis was more frequent in the study group. ${ }^{24}$ This is usually associated with significant worsening of quality of life, given that it leads to limitations in manual activities. These data from patients who underwent operations were also observed in an epidemiological study conducted by our group in $2017 .{ }^{13}$ That study also showed that axillary hyperhidrosis ${ }^{25}$ was the second most frequent site, followed by cranial-facial and plantar sites. ${ }^{26,27}$

Table 5. Degree of satisfaction after the surgery

\begin{tabular}{lcc} 
& Patients \\
& & $\mathbf{n}=\mathbf{2 , 3 3 0}$ \\
Degree of postoperative & $100 \%$ & $1586(68.1 \%)$ \\
satisfaction & $90 \%$ & $543(23.3 \%)$ \\
[n (\%)] & $75 \%$ & $170(5.9 \%)$ \\
& $<50 \%$ & $31(1.3 \%)$ \\
\hline
\end{tabular}

In our sample, the best results were in relation to the palmar and axillary sites. More than $90 \%$ of the patients in this study reported achieving major clinical improvement. This corroborates the indication for sympathectomy.

It has been demonstrated that the higher the level of VATS ganglion resection is, the greater the incidence of severe compensatory hyperhidrosis will be. The incidence of severe $\mathrm{CH}$ will also be greater if more than one ganglion level is resected in the same surgery. ${ }^{6,28,29}$ In addition, with higher resections, we observed higher incidence of Horner's syndrome. Thus, we avoided extended resections in our group and gave preference to G4 or G3 ganglion sympathectomies, as demonstrated in this study, in which these were the most frequent. Earlier in our practice, resection was performed at G2 for palmar hyperhidrosis. Later on, we saved G2 in order to reduce complications. More recently, we have demonstrated that simple resection of $\mathrm{G} 4$ leads to therapeutic success similar to that observed with $\mathrm{G} 3$ resection but with a lower $\mathrm{CH}$ rate and maintenance of satisfaction indices with the procedure. ${ }^{30}$ Thus, we have shown that more inferior resections are equally effective and result in a lower possibility of side effects. The prevalence of severe compensatory hyperhidrosis was $22.1 \%$ in our sample, which was compatible with findings already reported in the literature. ${ }^{31-33}$

Success in surgical treatment of patients has been found to be independent of age, ${ }^{34}$ even in patients for whom there was previous clinical treatment failure. ${ }^{35}$ More than $95 \%$ of the patients reach moderate to high clinical improvement at the main site of hyperhidrosis.

Weng et al. retrospectively reviewed 506 patients with palmar hyperhidrosis who were treated with either $\mathrm{R} 4$ or $\mathrm{R} 4+5$, in order to evaluate the long-term results regarding postoperative moist hands (PMH) after sympathectomy. ${ }^{36} \mathrm{PMH}$ occurred in over half of the patients after sympathectomy, but most of these patients were satisfied with the surgical results. Only six patients (1.3\%) were dissatisfied because of frequent $\mathrm{PMH}$. Those findings are consistent with our data, which showed improved quality of life in most patients. Thus, we also believe the main objective of treatment should be to improve the patient's quality of life.

Several tools are available for assessing quality of life among hyperhidrosis patients. Wade et al. studied a wide diversity of tools. ${ }^{37}$ Twenty-two quality-of-life tools were identified. The most

Table 6. Correlation of technique with compensatory hyperhidrosis

\begin{tabular}{|c|c|c|c|c|c|c|}
\hline \multirow{2}{*}{ Technique } & \multicolumn{4}{|c|}{ Compensatory hyperhidrosis } & \multirow{2}{*}{ Total } & \multirow{2}{*}{$\mathbf{P}^{*}$} \\
\hline & Absent & Mild & Moderate & Severe & & \\
\hline G4 & $126(15.2 \%)$ & 331 (39.9\%) & $249(30 \%)$ & $122(14.7 \%)$ & 828 & \\
\hline G3 G4 & 29 (10.9\%) & $62(23.4 \%)$ & $96(36.3 \%)$ & 77 (29.1\%) & 264 & \\
\hline G3 & $107(12.7 \%)$ & $271(32.3 \%)$ & $310(36.9 \%)$ & $150(17.9 \%)$ & 838 & $<0.00001$ \\
\hline G2 G3 & $42(11.5 \%)$ & $53(14.5 \%)$ & $140(38.3 \%)$ & $130(35.6 \%)$ & 365 & \\
\hline G2 & $7(7 \%)$ & $20(20 \%)$ & $36(36 \%)$ & $37(37 \%)$ & 100 & \\
\hline
\end{tabular}

"Chi-square test; $\mathrm{G}=$ ganglion. 
commonly used tools were the Hyperhidrosis Disease Severity Scale, the Dermatology Quality of Life Index and the Hyperhidrosis Quality-of-Life Questionnaire.

All the patients operated under our care presented poor or very poor quality of life and were preoperatively counselled regarding the risk of surgical failure and the incidence of severe compensatory hyperhidrosis after VATS. During the postoperative period, we observed improvement in the quality of life in more than $90 \%$ of the patients, and the degree of satisfaction was considered excellent or good in more than $90 \%$ of this sample. In addition, there was significant clinical improvement (greater than 90\%) when the main site of hyperhidrosis was the hands or axilla. These findings reflect the patients' expectations before and after the procedure, and demonstrate that VATS is a therapeutic method that can have a marked positive impact on the lives of patients with $\mathrm{PH}$ who seek treatment.

\section{CONCLUSIONS}

Bilateral VATS is a therapeutic method that decreases the degree of sweating in more than $90 \%$ of the patients with palmar and axillary hyperhidrosis. It improves the quality of life of more than $90 \%$ of the patients, at the expense of development of $\mathrm{CH}$ in approximately $90 \%$ of patients, but not intensely.

\section{REFERENCES}

1. Cameron AE. Selecting the Right Patient for Surgical Treatment of Hyperhidrosis. Thorac Surg Clin. 2016;26(4):403-6. PMID: 27692198; https://doi.org/10.1016/j.thorsurg.2016.06.004.

2. Wolosker N, de Campos JR, Kauffman P, et al. Evaluation of quality of life over time among 453 patients with hyperhidrosis submitted to endoscopic thoracic sympathectomy. J Vasc Surg. 2012;55(1):154-6. PMID: 22047833; https://doi.org/10.1016/j.jvs.2011.07.097.

3. Cerfolio RJ, de Campos JR, Bryant AS, et al. The Society of Thoracic Surgeons Expert Consensus for the Surgical Treatment of Hyperhidrosis. Ann Thorac Surg. 2011;91(5):1642-8. PMID: 21524489; https://doi. org/10.1016/j.athoracsur.2011.01.10.

4. Dias LI, Miranda EC, Toro IF, Mussi RK. Relationship between anxiety, depression and quality of life with the intensity of reflex sweating after thoracoscopic sympathectomy for treatment of primary hyperhidrosis. Rev Col Bras Cir. 2016;43(5):354-9. PMID: 27982329; https://doi. org/10.1590/0100-69912016005013.

5. Dharmaraj B, Kosai NR, Gendeh H, Ramzisham AR, Das S. A Prospective Cohort Study on Quality of Life after Endoscopic Thoracic Sympathectomy for Primary Hyperhidrosis. Clin Ter. 2016;167(3):67-71. PMID: 27424505; https://doi.org/10.7417/CT.2016.1928.

6. OngW,LeeA,TanWB,Lomanto D. Long-term results of a randomized controlled trial of T2 versus T2-T3 ablation in endoscopic thoracic sympathectomy for palmar hyperhidrosis. Surgical Endoscopy. 2016;30(3):1219-25. PMID: 26150222; https://doi.org/10.1007/s00464-015-4335-2.
7. Teivelis MP, Varella AY, Wolosker N. Expanded level of sympathectomy and incidence or severity of compensatory hyperhidrosis. J Thorac Cardiovasc Surg. 2014;148(5):2443-4. PMID: 25444213; https://doi. org/10.1016/j.jtcvs.2014.08.025.

8. Wolosker N, Yazbek G, de Campos JRM, et al. Quality of life before surgery is a predictive factor for satisfaction among patients undergoing sympathectomy to treat hyperhidrosis. JVasc Surg. 2010;51(5):1190-4. PMID: 20299178; https://doi.org/10.1016/j.jvs.2009.11.078.

9. Lee SS, Lee YU, Lee J-H, Lee JC. Comparison of the Long-Term Results of R3 and R4 Sympathicotomy for Palmar Hyperhidrosis. Korean $J$ Thorac Cardiovasc Surg. 2017;50(3):197-201. PMID: 28593156; https:// doi.org/10.5090/kjtcs.2017.50.3.197.

10. Amir M, Arish A, Weinstein Y, Pfeffer M, Levy Y. Impairment in quality of life among patients seeking surgery for hyperhidrosis (excessive sweating): preliminary results. Isr J Psychiatry Relat Sci. 2000;37(1):2531. PMID: 10857268.

11. Campos JRM de, Kauffman P, Werebe E de C, et al. Questionnaire of quality of life in patients with primary hyperhidrosis. Jornal de Pneumologia. 2003;29(4):178-81.

12. de Campos JRM, Kauffman P, Werebe E de C, et al. Quality of life, before and after thoracic sympathectomy: report on 378 operated patients. Ann Thorac Surg. 2003;76(3):886-91. PMID: 12963223; https://doi. org/10.1016/s0003-4975(03)00895-6.

13. Estevan FA, Wolosker MB, Wolosker N, Puech-Leão P. Epidemiologic analysis of prevalence of the hyperhidrosis. An Bras Dermatol. 2017;92(5):630-4. PMID: 29166497; https://doi.org/10.1590/abd18064841.20175551

14. Wolosker N, Krutman M, Kauffman P, et al. Effectiveness of oxybutynin for treatment of hyperhidrosis in overweight and obese patients. Rev Assoc Med Bras (1992). 2013;59(2):143-7. PMID: 23582555; https://doi. org/0.1016/j.ramb.2012.11.002

15. Wolosker N, de Campos JRM, Kauffman P, et al. Use of oxybutynin for treating plantar hyperhidrosis. Int J Dermatol. 2013;52(5):620-3. PMID: 23590378; https://doi.org/10.1111/j.1365-4632.2012.05746.x.

16. Wolosker N, Teivelis MP, Krutman M, et al. Long-Term Efficacy of Oxybutynin for Palmar and Plantar Hyperhidrosis in Children Younger than 14 Years. Pediatr Dermatol. 2015;32(5):663-7. PMID: 25490865; https://doi.org/10.1111/pde.12385

17. de Moura Júnior NB, das-Neves-Pereira JC, de Oliveira FRG, et al. Expression of acetylcholine and its receptor in human sympathetic ganglia in primary hyperhidrosis. Ann Thorac Surg. 2013;95(2):465-70. PMID: 23219256; https://doi.org/10.1016/j.athoracsur.2012.10.068

18. Wolosker N, Schvartsman C, Krutman M, et al. Efficacy and quality of life outcomes of oxybutynin for treating palmar hyperhidrosis in children younger than 14 years old. Pediatr Dermatol. 2014;31(1):48-53. PMID: 23627681; https://doi.org/10.1111/pde.12142.

19. Haider A, Solish N. Focal hyperhidrosis: diagnosis and management. CMAJ. 2005;172(1):69-75. PMID: 15632408; https://doi.org/10.1503/ cmaj.1040708. 
20. Sammons JE, Khachemoune A. Axillary hyperhidrosis: a focused review. J Dermatolog Treat. 2017;28(7):582-90. PMID: 28318360; https://doi.or g/10.1080/09546634.2017.1309347

21. Karimian-Teherani D, Panhofer P, Ringhofer C, et al. New epidemiological aspects of patients with severe hyperhidrosis presenting for sympathetic surgery. J Eur Acad Dermatol Venereol. 2009;23(6):651-5. PMID: 19250333; https://doi.org/10.1111/j.1468-3083.2009.03131.x.

22. Wolosker N, Krutman M, Campdell TPDA, et al. Oxybutynin treatment for hyperhidrosis: a comparative analysis between genders. Einstein (Sao Paulo). 2012;10(4):405-8. PMID: 23386077; https://doi.org/10.1590/ s1679-45082012000400002.

23. de Campos JRM, Wolosker N, Takeda FR, et al. The body mass index and level of resection: predictive factors for compensatory sweating after sympathectomy. Clin Auton Res. 2005;15(2):116-20. PMID: 15834768; https://doi.org/10.1007/s10286-005-0259-6.

24. Teivelis MP, Wolosker N, Krutman M, et al. Treatment of uncommon sites of focal primary hyperhidrosis: experience with pharmacological therapy using oxybutynin. Clinics (Sao Paulo). 2014;69(9):608-14. PMID: 25318092; https://doi.org/10.6061/clinics/2014(09)06.

25. Wolosker N, Teivelis MP, Krutman M, et al. Long-term results of the use of oxybutynin for the treatment of axillary hyperhidrosis. Ann Vasc Surg. 2014;28(5):1 106-12. PMID: 24512855; https://doi.org/10.1016/j. avsg.2013.12.024.

26. Wolosker $\mathrm{N}$, Teivelis MP, Krutman M, et al. Long-term results of oxybutynin treatment for palmar hyperhidrosis. Clin Auton Res. 2014;24(6):297-303. PMID: 25427685; https://doi.org/10.1007/s10286-014-0264-8.

27. Wolosker N, Teivelis MP, Krutman M, et al. Long-term results of oxybutynin use in treating facial hyperhidrosis. An Bras Dermatol. 2014;89(6):912-6. PMID: 25387496; https://doi.org/10.1590/abd1806-4841.20143272.

28. Ribas Milanez de Campos J, Kauffman P, Wolosker N, et al. Axillary hyperhidrosis: T3/T4 versus T4 thoracic sympathectomy in a series of 276 cases. J Laparoendosc Adv Surg Tech A. 2006;16(6):598-603. PMID: 17243877; https://doi.org/10.1089/lap.2006.16.598.

29. Yazbek G, Wolosker N, Milanez de Campos JR, et al. Palmar hyperhidrosiswhich is the best level of denervation using video-assisted thoracoscopic sympathectomy:T2 orT3 ganglion? J Vasc Surg. 2005;42(2):281-5. PMID: 16102627; https://doi.org/10.1016/j.jvs.2005.03.041.

30. Milanez de Campos JR, Kauffman P, Gomes O, Wolosker N. Video-Assisted Thoracic Sympathectomy for Hyperhidrosis. Thorac Surg Clin. 2016;26(3):34758. PMID: 27427529; https://doi.org/10.1016/j.thorsurg.2016.04.010.

31. Teivelis MP, Wolosker N, Krutman M, et al. Compensatory hyperhidrosis: results of pharmacologic treatment with oxybutynin. Ann Thorac Surg. 2014;98(5):1797-802. PMID:25173719; https://doi.org/10.1016/j. athoracsur.2014.05.087.

32. Vannucci F, Araújo JA. Thoracic sympathectomy for hyperhidrosis: from surgical indications to clinical results. J Thorac Dis.
2017;9(Suppl 3):S178-92. PMID: 28446983; https://doi.org/10.21037/ jtd.2017.04.04.

33. de Andrade Filho LO, Kuzniec S, Wolosker N, et al. Technical difficulties and complications of sympathectomy in the treatment of hyperhidrosis: an analysis of 1731 cases. Ann Vasc Surg. 2013;27(4):447-53. PMID: 23406790; https://doi.org/10.1016/j.avsg.2012.05.026.

34. Leiderman DBD, Milanez de Campos JR, Kauffman P, et al. The relation between age and outcomes of thoracic sympathectomy for hyperhidrosis: The older the better. JThorac Cardiovasc Surg. 2018;156(4):1748-56. PMID: 30054140; https://doi.org/10.1016/j.jtcvs.2018.05.084.

35. Lembrança L, Wolosker N, de Campos JRM, et al. Videothoracoscopic Sympathectomy Results after Oxybutynin Chloride Treatment Failure. Ann Vasc Surg. 2017:43:283-7. PMID: 28478174; https://doi.org/10.1016/j. avsg.2017.01.018.

36. Weng W, Liu Y, Li J, et al. A long-term evaluation of postoperative moist hands after R4/R4+5 sympathectomy for primary palmar hyperhidrosis. JThorac Dis. 2018;10(3):1532-7. PMID:29707303; https:// doi.org/10.21037/jtd.2018.02.07.

37. Wade R, Jones-Diette J, Wright K, Layton AM, Woolacott N. Hyperhidrosis quality of life measures: review and patient perspective. J Dermatolog Treat. 2019;30(3):303-8. PMID: 30051732; https://doi.org/10.1080/095 46634.2018.1506080.

Authors' contributions: Wolosker N; de Campos JRM; Kauffman P; da Silva MFA, Faustino CB; Tedde ML; Puech-Leão P and Pêgo-Fernandes PM: conceptualization (equal), data curation (equal), formal analysis (equal), funding acquisition (equal), investigation (equal), methodology (equal), project administration (equal), resources (equal), software (equal), supervision (equal), validation (equal), visualization (equal), writing-original draft (equal) and writing-review and editing (equal)

\section{Sources of funding: None}

Conflict of interest: None

Date of first submission: January 28, 2021

Last received: June 9, 2021

Accepted: July 23, 2021

\author{
Address for correspondence: \\ Marcelo Fiorelli Alexandrino da Silva \\ Av Albert Einstein, 627 \\ São Paulo (SP) — Brasil \\ CEP 05652-000 \\ Tel. (+55 11) 2151-5015 \\ Fax. (+55 11) 3885-5361 \\ E-mail:marcelo.fiorelli96@gmail.com
}

